\title{
Understanding the interaction of soft and hard magnetic components in NdFeB with first-order reversal curves
}

\author{
Sven Erik Ilse $\odot,{ }^{*}$ Felix Groß $\odot$, Gisela Schütz, Joachim Gräfe $\odot$, and Eberhard Goering ${ }^{\dagger}$ \\ Max Planck Institute for Intelligent Systems, Stuttgart, Germany
}

\begin{abstract}
(Received 15 October 2020; revised 16 December 2020; accepted 24 December 2020; published 15 January 2021)
\end{abstract}
\begin{abstract}
First-order reversal curve (FORC) measurements are a powerful tool to study magnetization reversal processes and interactions in heterogeneous systems with broad coercivity distributions. In $\mathrm{NdFeB}$ hard magnets an additional soft magnetic component is often observed possibly originating from damaged surface grains. Here we use FORC to study the reversal processes and interactions in these permanent magnets at different temperatures between 50 and $350 \mathrm{~K}$. The measured reversal curves reveal a strongly coupled switching of the soft and hard magnetic components above $250 \mathrm{~K}$. Below this temperature the two components are decoupled and switch almost independently. This decrease in effective interaction at lower temperatures is also observed in the FORC diagrams by a relative reduction in intensity of the so called interaction peak. This result proves that FORC is a powerful method, contributing to a better understanding of magnetization reversal processes and interactions in permanent magnets.
\end{abstract}

DOI: 10.1103/PhysRevB.103.024425

\section{INTRODUCTION}

First-order reversal curves (FORCs) can be utilized to study magnetic properties which are inaccessible for conventional magnetic characterization techniques. Measuring hysteresis loops with magneto-optic Kerr effect (MOKE) microscopy, a superconducting quantum interference device (SQUID), or a conventional magnetometer give information about ensemble related properties such as coercive and saturation field, remanence, and saturation magnetization. FORC, however, is able to provide more elaborate information such as coercive and interaction field distributions or interaction strength between interacting magnetic components [1,2]. Another advantage of FORC is that it can yield microstructural information about a system without the need of actual lateral resolution $[2,3]$.

FORC has been used to study a variety of different systems as geological and biogenic samples [4-6], microstructured and model magnetic systems $[2,7,8]$, permanent hard magnetic systems [9-15], or soft magnetic nanowires [3].

The FORC technique originates from the Preisach model of hysteresis, developed in 1935, for which all coercivities and interactions within a system are considered [16]. In 1986 Mayergoyz proposed to use first-order reversal curves as an experimental approach to the theoretical Preisach distribution. However, as a necessary condition a system has to meet

\footnotetext{
*ilse@is.mpg.de

${ }^{\dagger}$ goering@is.mpg.de
}

Published by the American Physical Society under the terms of the Creative Commons Attribution 4.0 International license. Further distribution of this work must maintain attribution to the author(s) and the published article's title, journal citation, and DOI. Open access publication funded by the Max Planck Society. the wiping-out and congruency property such that the FORC distribution represents the Preisach distribution [17]. Real systems often violate at least one of these properties which is why a FORC distribution cannot be naively interpreted as the Preisach distribution [18]. For this reason FORC diagrams are often not thoroughly understood but rather used as a magnetic fingerprint [19-21]. However, a lot of work, especially on simplified model systems, has been done leading to a better and more quantitative understanding of various characteristic FORC peaks. FORC peaks arising from kinetic effects [22], wishbone shaped FORC diagrams [1], or interaction peaks caused by interaction between magnetic components with different coercivities [2] are nowadays well understood.

In this work we are going to study a real system, namely NdFeB samples, instead of a simplified model system. Previous studies revealed that $\mathrm{NdFeB}$ permanent magnets can consist of distinct soft and hard magnetic components [9-11,14,23,24]. Hence, it is an interesting material to study the interaction between the soft and hard magnetic components with FORC. In a previous work we studied such an interaction using FORC in a model system and varied the interaction strength by geometrically alternating the system [2]. The interaction in NdFeB samples cannot be varied that easily without changing the magnetic properties of the whole sample. Magnetic properties strongly depend on the microstructure of the samples and even samples cut from the same bulk magnet might show variations. Thus, we measure FORCs at different temperatures to study temperature related variations of the effective interaction between soft and hard magnetic components and the corresponding magnetization reversal processes. Using temperature as a parameter to vary the effective interaction and performing all measurements on the same sample allows us to systematically trace back all FORC peak variations without the need of a extensive microstructural analysis of different samples. 


\section{EXPERIMENTAL METHODS}

The studied hard magnets are sintered, commercially available neodymium-iron-boron magnets, purchased at [25] in the shape of a $10 \times 10 \times 10 \mathrm{~mm}^{3}$ cube with a nickel coating and a magnet grade of N42. The cube was cut into rods with a square base of $1 \mathrm{~mm}$ and a length of $2 \mathrm{~mm}$ via wire electrical discharge machining. The easy axis lies along the long edge of the rod. Since the demagnetizing factor depends on the geometry of the samples the shape of the hysteresis loop and the FORC density will change for different geometries. To obtain geometry independent results a demagnetizing correction for each minor loop of a FORC measurement has to be applied. Here we also study the influence of demagnetizing fields to the FORC density and compare raw data with demagnetizing corrected data. For this correction the total field $H_{\text {tot }}$ has to be calculated:

$$
H_{\mathrm{tot}}=H_{\mathrm{appl}}-H_{\mathrm{d}}=H_{\mathrm{appl}}-N_{\mathrm{d}} M,
$$

with the applied field $H_{\text {appl }}$, the demagnetizing field $H_{\mathrm{d}}$, the samples magnetization $M$, and the geometry dependent demagnetizing factor $N_{\mathrm{d}}$. Sato and Ishii [26] showed that the demagnetizing factor $N_{\mathrm{d}}$ along the long edge of a rod can be approximated with

$$
N_{\mathrm{d}}=\frac{1}{2 n+1},
$$

where $n$ is the ratio of long to short rod edge. With a ratio of $n=2$ the measured NdFeB sample has a demagnetizing factor of $N_{\mathrm{d}}=0.2$.

For the magnetic measurements a Quantum Design MPMS 3 SQUID magnetometer is used. An in-house written software automatizes the measurement of first-order reversal curves and subsequently analyzes the data. With this setup we are able to measure a detailed set of FORC on hard, permanent magnets such as $\mathrm{NdFeB}$ within 15 to $20 \mathrm{~h}$ at temperatures between 5 and $1000 \mathrm{~K}$ and with an applied field of up to $7 \mathrm{~T}$. A FORC measurement consists of a set of minor hysteresis loops, where each loop starts at a different reversal field $H_{\mathrm{r}}$ and is measured until positive saturation $H_{\text {sat }}$. Figure 1 illustrates the applied field cycle for one single minor loop measurement, which is repeated until the desired number of FORCs within the field range $-H_{\text {sat }}<H_{\mathrm{r}}<H_{\text {sat }}$ are measured. To determine the FORC density the mixed second derivative of the measured magnetization surface $M\left(H_{\text {appl }}, H_{\mathrm{r}}\right)$ has to be calculated:

$$
\rho\left(H_{\mathrm{appl}}, H_{\mathrm{r}}\right)=-\frac{1}{2} \frac{\partial^{2} M\left(H_{\mathrm{appl}}, H_{\mathrm{r}}\right)}{\partial H_{\mathrm{appl}} \partial H_{\mathrm{r}}} .
$$

A subsequent transformation of the $H_{\text {appl }}$ and $H_{\mathrm{r}}$ axis into a coercive field $H_{\mathrm{c}}$ and an interaction field $H_{\mathrm{u}}$ axis simplifies the interpretation of a FORC density:

$$
H_{\mathrm{c}}=\frac{1}{2}\left(H_{\mathrm{appl}}-H_{\mathrm{r}}\right), \quad H_{\mathrm{u}}=\frac{1}{2}\left(H_{\mathrm{appl}}+H_{\mathrm{r}}\right) .
$$

For a more detailed introduction into the FORC method the reader is referred to $[1,6,27]$. The calculation of the mixed second derivative to obtain the FORC densities was done with the gFORC algorithm [28].

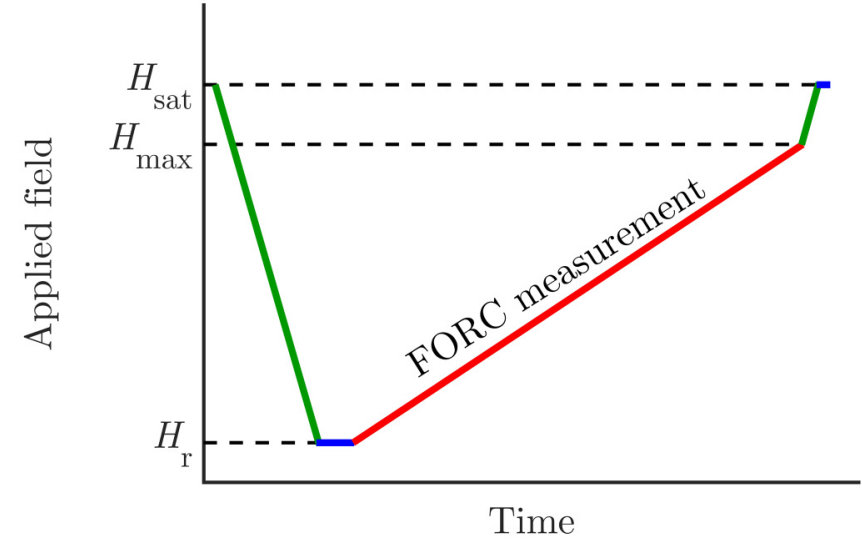

FIG. 1. Applied field over time for one single first-order reversal curve measurement. The segments where the field is driven from saturation $H_{\text {sat }}$ to reversal field $H_{\mathrm{r}}$ and from $H_{\text {max }}$ to $H_{\text {sat }}$ are displayed in green, the actual measurement of a FORC in red, and short waiting times in blue.

\section{RESULTS}

\section{A. Effect of demagnetization correction on FORC densities}

Figure 2 shows the room temperature hysteresis loop of the $\mathrm{NdFeB}$ sample with and without demagnetizing correction. The magnetization reversal happens in two steps indicating the soft and hard magnetic component the sample consists of. Such soft magnetic components in $\mathrm{NdFeB}$ magnets have been observed in many previous works [9-11,24] and may have its origin in damaged surface grains due to the cutting process $[14,15,23]$. The soft and hard magnetic components related room temperature coercive fields could be estimated to $H_{\mathrm{c} \text {, soft }}=0.1 \mathrm{~T}$ and $H_{\mathrm{c} \text {,hard }}=1.25 \mathrm{~T}$, respectively, after the demagnetization correction. In saturation the demagnetizing

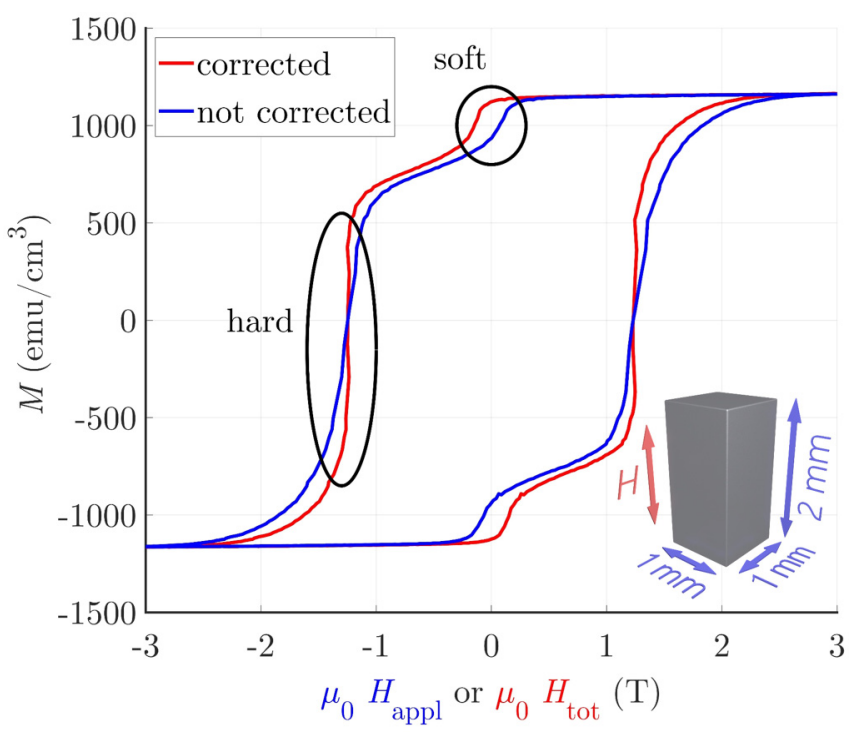

FIG. 2. Hysteresis loop at $300 \mathrm{~K}$ of sintered, commercial NdFeB magnet with (red) and without (blue) demagnetizing correction. The soft and hard magnetic switching regions are marked by ellipses. The sample geometry is shown in the inset. 

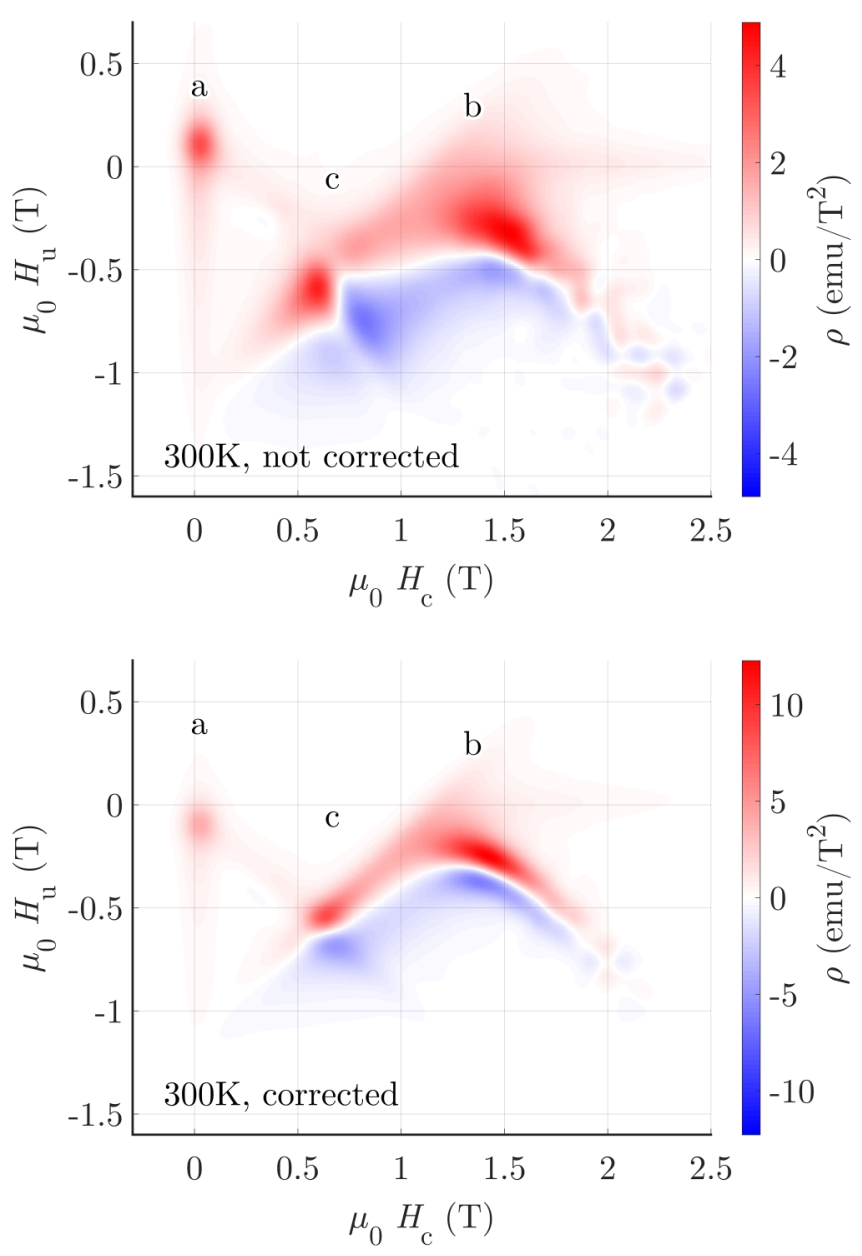

FIG. 3. NdFeB FORC densities at $300 \mathrm{~K}$ without (top) and with (bottom) demagnetization correction. Positive FORC peaks are shown in red, negative peaks in blue. Three characteristic features are visible: (a) and (b) switching of soft and hard magnetic components, respectively, and (c) interaction peak.

field is at its maximum and has, according to Eq. (1), a value of $H_{\mathrm{d}}=0.26 \mathrm{~T}$. After the soft magnetic component switches the remaining demagnetizing field has a value of $H_{\mathrm{d}}=0.2 \mathrm{~T}$.

Figure 3 displays the demagnetizing field uncorrected (top) and corrected (bottom) FORC diagram. Three prominent features are distinguishable. Peak (a) corresponds to the switching of the soft magnetic component in the sample, peak (b) arises from the switching of the hard magnetic components, and peak (c) is the so called interaction peak [2]. The demagnetization correction can be seen as a correction for the samples interaction with its own global stray field. By correcting each minor loop of a FORC measurement and comparing the resulting FORC diagram to the uncorrected one we can study how this interaction affects the FORC diagram. Both FORC diagrams in Fig. 3 show the same three prominent features. However, the peaks in the demagnetization corrected FORC density are much narrower in interaction field $H_{\mathrm{u}}$ direction and more pronounced. Hence, the interaction of the sample with its own stray field is related to a $H_{\mathrm{u}}$ broadened FORC density. Additionally, the soft magnetic peak position (a) shifts from $H_{\mathrm{u}}=0.09 \mathrm{~T}$ without correction

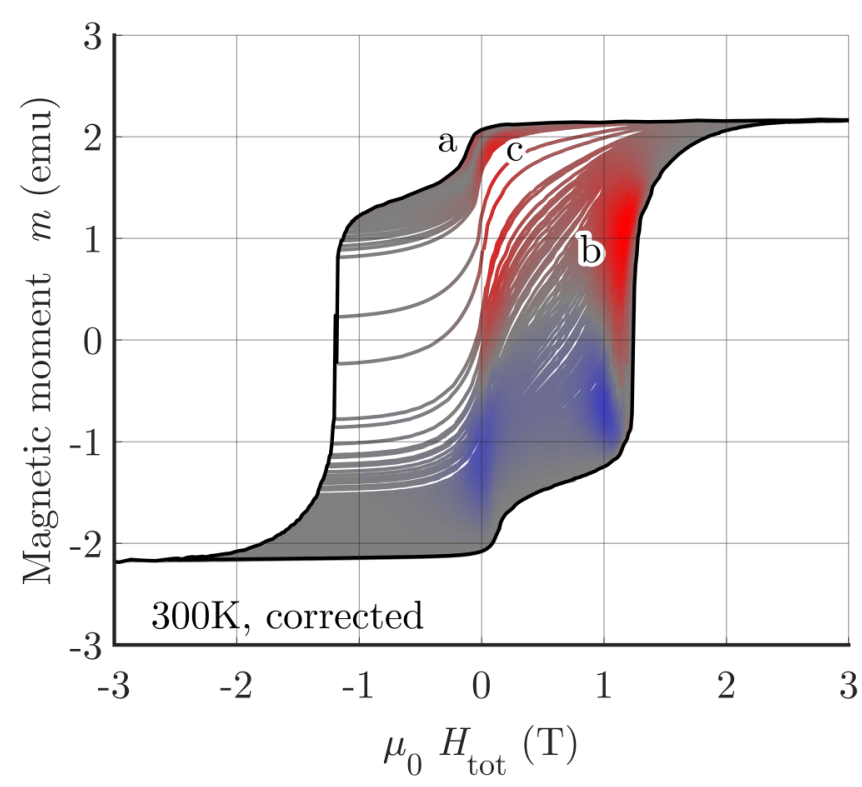

FIG. 4. Demagnetizing field corrected FORC density at room temperature projected onto the minor loops. The origin of the three FORC peaks visible in Fig. 3 is again labeled with (a), (b), and (c).

to $H_{\mathrm{u}}=-0.11 \mathrm{~T}$ with demagnetizing correction. This total shift of $0.2 \mathrm{~T}$ is caused by the demagnetizing field $H_{\mathrm{d}}=0.2 \mathrm{~T}$ after the soft magnetic component has switched. Nevertheless, no extra peaks appear and the characteristic peaks are distinguishable with and without correction.

To understand the origin of the individual peaks we project the FORC density by simultaneous color coding onto the minor hysteresis loops, shown in Fig. 4. For further details on how the projection is done see [28]. Peaks (a) and (b) originate from the switching of the soft and hard magnetic components, respectively. The interaction peak (c) results from an interaction between soft and hard magnetic components. Hard magnetic components switch back along with soft magnetic components close to zero applied field, even though a field of around $-1.25 \mathrm{~T}$ is needed to switch them in the first place. Since this switching happens at applied fields around $H_{\text {appl }}=H_{\mathrm{c} \text {,soft }}$, and in minor loops which start at reversal fields around the coercive field of the hard magnetic component $H_{\mathrm{r}}=-H_{\mathrm{c}, \text { hard }}$, the coordinates of the interaction peak can be calculated as [2]

$$
H_{\mathrm{u}}=\frac{1}{2}\left(H_{\mathrm{c}, \text { soft }}-H_{\mathrm{c}, \text { hard }}\right)
$$

and

$$
H_{\mathrm{c}}=\frac{1}{2}\left(H_{\mathrm{c}, \text { soft }}+H_{\mathrm{c}, \text { hard }}\right)
$$

\section{B. Temperature dependent FORC densities}

In order to study how the coupling changes with temperature several FORC diagrams between 50 and $350 \mathrm{~K}$ in $50 \mathrm{~K}$ steps have been measured. The FORC diagrams in Fig. 5 show that the coercive field of the hard magnetic component increases with decreasing temperature. At $350 \mathrm{~K}$ it has a value of $H_{\mathrm{c} \text {,hard }} \approx 1 \mathrm{~T}$ and increases to $H_{\mathrm{c} \text {,hard }} \approx 4.5 \mathrm{~T}$ at $50 \mathrm{~K}$. The coercivity of the soft magnetic component remains almost unchanged over the whole temperature range at $H_{\mathrm{c} \text {,soft }} \approx 0.1 \mathrm{~T}$. 

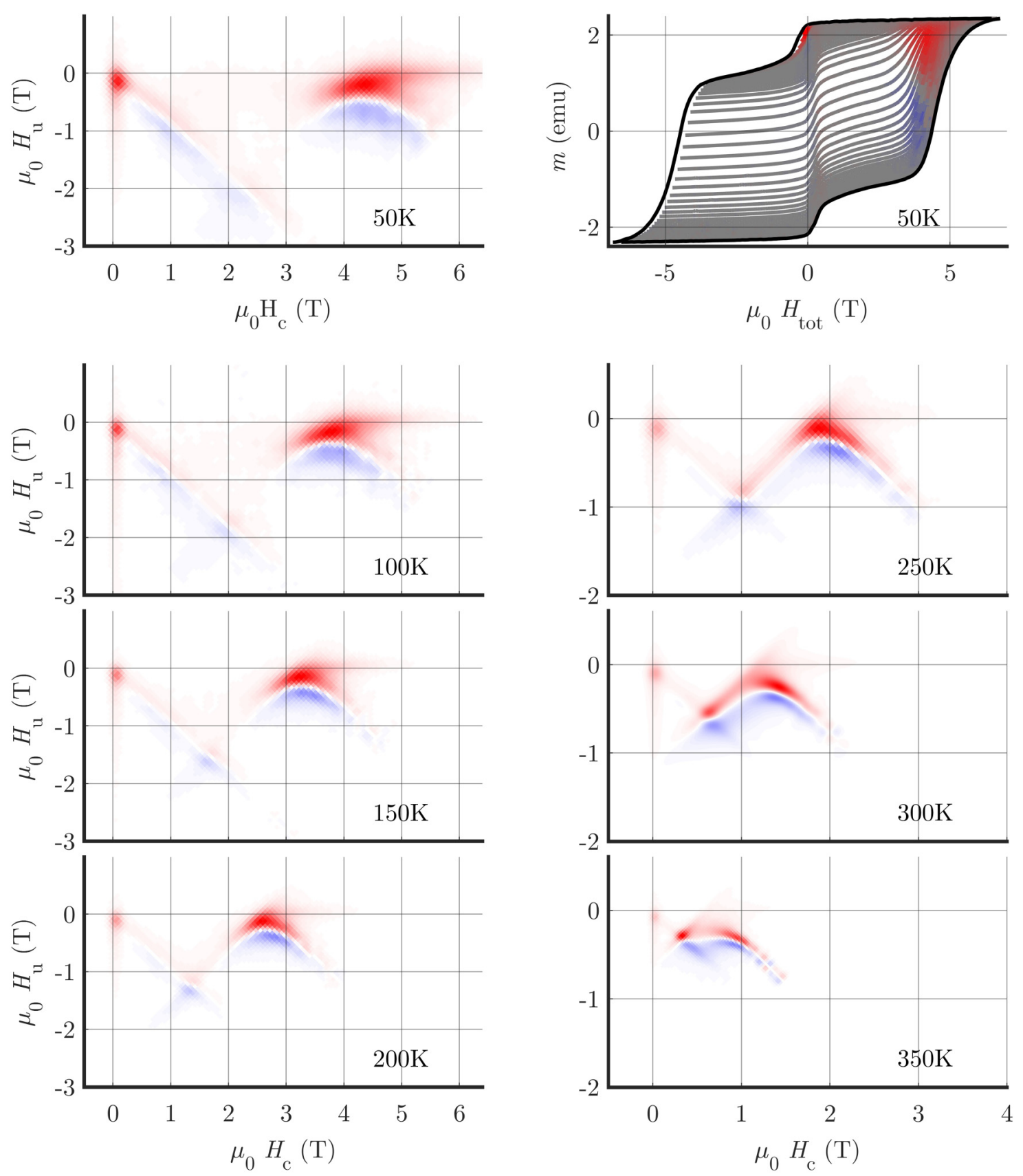

FIG. 5. NdFeB FORC densities between 50 and $350 \mathrm{~K}$. Top left: The $50 \mathrm{~K}$ FORC density is projected onto the minor loops. Note the axis scaling in the left and right column is not the same for a better visibility of the FORC densities.

The position of the interaction peak follows the coordinates calculated with Eqs. (5) and (6). The intensity of the interaction peak (c) decreases with temperature and almost vanishes at $50 \mathrm{~K}$. In Fig. 6 the relative intensities of the hard, soft, and interaction peak are plotted over temperature. The relative intensities are obtained by integrating over the positive (red) component of a peak and dividing it by the integral over the whole FORC diagram. Since the integration over the whole FORC diagram results in the saturation magnetization of the sample [27] the integration over the soft and hard magnetic peak is related to the saturation magnetization of the soft and hard magnetic component, respectively. The relative intensities of the soft and hard magnetic peaks are almost constant over the whole temperature range which indicates that the relative amounts of soft and hard magnetic material stays the same. The reason for the lower relative intensity of the hard magnetic peak at lower temperatures is that the peak stretches out above $7 \mathrm{~T}$ which could not be measured and therefore it is missing in the FORC density. As previously shown [2], the relative intensity of the interaction peak is a measure for the effective interaction strength. The relative intensity of the interaction peak of the $\mathrm{NdFeB}$ sample in Fig. 6 is almost constant and close to zero below $250 \mathrm{~K}$ but drastically increases above this temperature. Comparing the FORC density projected onto the minor loops at $300 \mathrm{~K}$ in Fig. 4 and at $50 \mathrm{~K}$ in Fig. 5 reveals that at $50 \mathrm{~K}$ the 


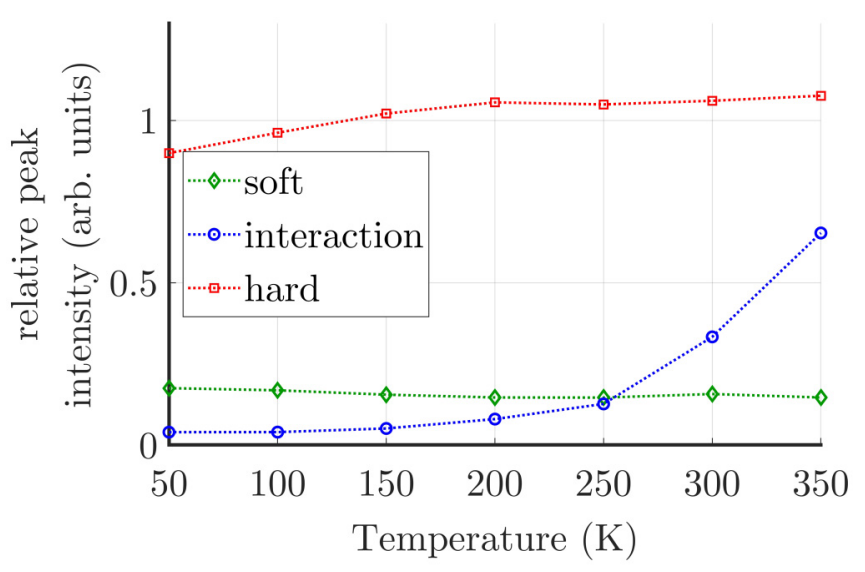

FIG. 6. Relative intensities of the three FORC peaks over temperature.

soft and hard magnetic components switch independently in each minor loop. At $50 \mathrm{~K}$ neighboring minor loops have the same slope around $H_{\text {tot }}=0 \mathrm{~T}$ and the magnetization reversal happens in two distinct steps which shows that soft and hard magnetic components are effectively decoupled. Thus, no interaction peak appears in the FORC density and only the soft magnetic (a) and hard magnetic peaks (b) are visible. However, at $300 \mathrm{~K}$ neighboring minor loops have different slopes around $H_{\mathrm{tot}}=0 \mathrm{~T}$ and the magnetization reversal for minor loops which start around the coercive field of the hard magnetic component $H_{\mathrm{r}}=-H_{\mathrm{c} \text {,hard }}$ does not happen in two distinct steps. Instead the soft and hard magnetic components are coupled and switch together in one reversal process which leads to the interaction peak (c) in the FORC density. The effective decoupling of soft and hard magnetic components at lower temperatures is caused by the highly increased coercive field $H_{\mathrm{c} \text {, hard }}$. As a result, the coupling between the two components is not strong enough to cause a simultaneous switching of soft and hard magnetic parts, effectively decoupling them. With increasing temperature the coercive field $H_{\mathrm{c} \text {, hard }}$ decreases, thus the coupling is strong enough to cause a partly simultaneous switching of soft and hard magnetic components. This decrease of effective interaction between soft and hard magnetic components in $\mathrm{NdFeB}$ magnets at lower temperatures appears as a relative intensity decrease of the interaction peak in the FORC density which can be taken as a measure for the effective coupling strength. The effect of decoupling at lower temperatures was observed for various $\mathrm{NdFeB}$ samples, including one with a different ratio of soft to hard magnetic component due to annealing. For measurement results on these additional samples see the Supplemental Material [29].

\section{SUMMARY}

The interaction between soft and hard magnetic components in commercial $\mathrm{NdFeB}$ samples has been studied using FORC measurements. First, room temperature FORC measurements with and without demagnetizing correction were compared. It appears that the samples interaction with its own stray field broadens the features in a FORC density along the $H_{\mathrm{u}}$ direction and shifts the soft magnetic peak in negative $H_{\mathrm{u}}$ direction. This broadening can be corrected by performing a demagnetizing correction for each measured minor loop. Before and after the correction the same three characteristic FORC peaks, (a) switching of the soft magnetic component, (b) switching of the hard magnetic component, and (c) interaction peak, are distinguishable. Measuring FORC densities at temperatures between 50 and $350 \mathrm{~K}$ revealed that the interaction peak almost vanishes for low temperatures. Projecting the FORC density onto the minor loops revealed that the switching of soft and hard magnetic components at low temperatures is effectively decoupled. By using FORC we were able to better understand the interactions and reversal processes in $\mathrm{NdFeB}$. Additionally, we showed that the relative intensity of the interaction peak can be used as a measure for the effective interaction strength not only in model systems [2] but in real systems as well.

\section{ACKNOWLEDGMENT}

The authors thankfully acknowledge Thomas Meisner and Arnold Weible for cutting the samples.
[1] C.-I. Dobrotă and A. Stancu, What does a first-order reversal curve diagram really mean? A study case: Array of ferromagnetic nanowires, J. Appl. Phys. 113, 043928 (2013).

[2] F. Groß, S. E. Ilse, G. Schütz, J. Gräfe, and E. Goering, Interpreting first-order reversal curves beyond the Preisach model: An experimental permalloy microarray investigation, Phys. Rev. B 99, 064401 (2019).

[3] F. Béron, L. Clime, M. Ciureanu, D. Ménard, R. W. Cochrane, and A. Yelon, First-order reversal curves diagrams of ferromagnetic soft nanowire arrays, IEEE Trans. Magnet. 42, 3060 (2006).

[4] L. Wetter, K. Verosub, and J. Russell, Detection of silicamediated dissolution of magnetic grains in sediments using FORC diagrams, Geophys. Res. Lett. 34, L12707 (2007).
[5] A. P. Chen, R. Egli, and B. M. Moskowitz, First-order reversal curve (FORC) diagrams of natural and cultured biogenic magnetic particles, J. Geophys. Res. 112, B08S90 (2007).

[6] A. P. Roberts, C. R. Pike, and K. L. Verosub, First-order reversal curve diagrams: A new tool for characterizing the magnetic properties of natural samples, J. Geophys. Res. 105, 28461 (2000).

[7] J. Gräfe, M. Weigand, C. Stahl, N. Träger, M. Kopp, G. Schütz, E. J. Goering, F. Haering, P. Ziemann, and U. Wiedwald, Combined first-order reversal curve and x-ray microscopy investigation of magnetization reversal mechanisms in hexagonal antidot lattices, Phys. Rev. B 93, 014406 (2016).

[8] J. Gräfe, M. Weigand, N. Träger, G. Schütz, E. J. Goering, M. Skripnik, U. Nowak, F. Haering, P. Ziemann, and U. Wiedwald, 
Geometric control of the magnetization reversal in antidot lattices with perpendicular magnetic anisotropy, Phys. Rev. B 93, 104421 (2016).

[9] H. Chiriac, N. Lupu, L. Stoleriu, P. Postolache, and A. Stancu, Experimental and micromagnetic first-order reversal curves analysis in NdFeB-based bulk "exchange spring"-type permanent magnets, J. Magn. Magn. Mater. 316, 177 (2007).

[10] T. Schrefl, T. Shoji, M. Winklhofer, H. Oezelt, M. Yano, and G. Zimanyi, First order reversal curve studies of permanent magnets, J. Appl. Phys. 111, 07A728 (2012).

[11] P. A. Chen, C. Y. Yang, S. J. Chang, M. H. Lee, N. K. Tang, S. C. Yen, and Y. C. Tseng, Soft and hard natures of $\mathrm{Nd}_{2} \mathrm{Fe}_{14} \mathrm{~B}$ permanent magnet explored by first-order-reversal-curves, J. Magn. Magn. Mater. 370, 45 (2014).

[12] T. Yomogita, S. Okamoto, N. Kikuchi, O. Kitakami, H. SepehriAmin, T. Ohkubo, K. Hono, T. Akiya, K. Hioki, and A. Hattori, Temperature and field direction dependences of first-order reversal curve (FORC) diagrams of hot-deformed Nd-Fe-B magnets, J. Magn. Magn. Mater. 447, 110 (2018).

[13] S. Muralidhar, J. Gräfe, Yu. C. Chen, M. Etter, G. Gregori, S. Ener, S. Sawatzki, K. Hono, O. Gutfleisch, H. Kronmüller, G. Schütz, and E. J. Goering, Temperature-dependent firstorder reversal curve measurements on unusually hard magnetic low-temperature phase of MnBi, Phys. Rev. B 95, 024413 (2017).

[14] K. Miyazawa, S. Okamoto, T. Yomogita, N. Kikuchi, O. Kitakami, K. Toyoki, D. Billington, Y. Kotani, T. Nakamura, T. Sasaki, T. Ohkubo, and K. Hono, First-order reversal curve analysis of a Nd-Fe-B sintered magnet with soft $\mathrm{x}$-ray magnetic circular dichroism microscopy, Acta Mater. 162, 1 (2019).

[15] S. Okamoto, K. Miyazawa, T. Yomogita, N. Kikuchi, O. Kitakami, K. Toyoki, D. Billington, Y. Kotani, T. Nakamura, T. Sasaki, T. Ohkubo, K. Hono, Y. Takada, T. Sato, Y. Kaneko, and A. Kato, Temperature dependent magnetization reversal process of a Ga-doped Nd-Fe-B sintered magnet based on first-order reversal curve analysis, Acta Mater. 178, 90 (2019).

[16] F. Preisach, Über die magnetische Nachwirkung, Z. Phys. 94, 277 (1935).
[17] I. D. Mayergoyz, Mathematical Models of Hysteresis, Phys. Rev. Lett. 56, 1518 (1986).

[18] C. R. Pike, A. P. Roberts, and K. L. Verosub, Characterizing interactions in fine magnetic particle systems using first order reversal curves, J. Appl. Phys. 85, 6660 (1999).

[19] B. F. Valcu, D. A. Gilbert, and K. Liu, Fingerprinting inhomogeneities in recording media using the first-order reversal curve method, IEEE Trans. Magn. 47, 2988 (2011).

[20] R. K. Dumas, C. P. Li, I. V. Roshchin, I. K. Schuller, and K. Liu, Magnetic fingerprints of sub-100 nm Fe dots, Phys. Rev. B 75, 134405 (2007).

[21] H. G. Katzgraber, F. Pázmándi, C. R Pike, K. Liu, R. T Scalettar, K. L. Verosub, and G. T. Zimányi, Reversal-Field Memory in the Hysteresis of Spin Glasses, Phys. Rev. Lett. 89, 257202 (2002).

[22] N. Siadou, M. Androutsopoulos, I. Panagiotopoulos, L. Stoleriu, A. Stancu, T. Bakas, and V. Alexandrakis, Magnetization reversal in $[\mathrm{Ni} / \mathrm{Pt}]_{6} / \mathrm{Pt}(\mathrm{x}) /[\mathrm{Co} / \mathrm{Pt}]_{6}$ multilayers, J. Magn. Magn. Mater. 323, 1671 (2011).

[23] K. Kobayashi, M. H. Nakamura, and K. Urushibata, Magnetic properties of the surface layer and its magnetic interaction with the interior of Nd-Fe-B sintered magnets, J. Appl. Phys. 117, 173909 (2015).

[24] H. Nakamura, K. Hirota, M. Shimao, T. Minowa, and M. Honshima, Magnetic properties of extremely small Nd-Fe-B sintered magnets, IEEE Trans. Magn. 41, 3844 (2005).

[25] https://www.supermagnete.de/

[26] M. Sato and Y. Ishii, Simple and approximate expressions of demagnetizing factors of uniformly magnetized rectangular rod and cylinder, J. Appl. Phys. 66, 983 (1989).

[27] C. Pike, First-order reversal-curve diagrams and reversible magnetization, Phys. Rev. B 68, 104424 (2003).

[28] F. Groß, J. C. Martínez-García, S. E. Ilse, G. Schütz, E. Goering, M. Rivas, and J. Gräfe, GFORC: A graphics processing unit accelerated first-order reversal-curve calculator, J. Appl. Phys. 126, 163901 (2019).

[29] See Supplemental Material at http://link.aps.org/supplemental/ 10.1103/PhysRevB.103.024425 for measurement results on additional samples showing the reproducibility of the results. 